\title{
PERFORMANCE ASSESMENT OF SPATIAL FILTERS IN NOISE REMOVAL BASED ON A DEGRADATION MODEL
}

\author{
S. Sukumaran \\ Dept. of Computer Science \\ Erode Arts \& Science College, Erode \\ Tamilnadu. India
}

\author{
M.Shanmugasundaram \\ Dept. of Computer Science \\ Erode Arts \& Science College, Erode \\ Tamilnadu. India
}

\begin{abstract}
The most significant feature of images is to reduce Gaussian noise which is commonly found in images and make better image quality. In recent years, technological development has significantly improved analyzing images. Spatial filtering is the method of choice in situations when only additive random noise is present. In this paper, an attempt is made to evaluate the performance of the four linear and non-linear filters used for removing noise from the image. The performance of the noise removal process is analyzed, and the results are compared with the previously reported.
\end{abstract}

\section{Keywords: spatial filters, Gaussian noise, linear and non linear filter, degradation}

\section{INTRODUCTION}

Filters are widely accepted to remove impulsive and high frequency noise for signal and image processing. The concept of filtering has its roots in the use of the Fourier transform for signal processing in the so-called frequency domain. Spatial filtering term [1] is the filtering operations that are performed directly on the pixels of an image. The process consists simply of moving the filter mask from point to point in an image at each point $(\mathrm{x}, \mathrm{y})$ and the response of the filter at that point is calculated using a predefined relationship. Linear and non-linear are two types of filters based on filtering process. In Linear spatial filtering, the result is the sum of products of the mask coefficients with the corresponding pixels directly under the mask.

$$
\begin{aligned}
& g\left(x_{w} y\right)=w(-1,-1) f(x-1, y-1)+ \\
& w(-1,0) f(x-1, y)+\cdots+ \\
& w(1,1) f(x+1, y+1)
\end{aligned}
$$

The coefficient $w(0,0)$ coincides with image value $f(x, y)$, indicating that the mask is centered at $(x, y)$ when the computation of sum of products takes place.

For a mask of size $m X n$, we assume that $m=2 a+1$ and $n=2 b+1$, where $a$ and $b$ are nonnegative integer. Then $m$ and $n$ are odd. In general, linear filtering of an image $f$ of size $\mathrm{MxN}$ with a filter mask of size mxn is given by the expression:

$g\left(x_{w} y\right)=\sum_{g=-a}^{a} \sum_{g=-b}^{b} w\left(s_{0} t\right) f\left(x+s_{v} y+t\right)$

The process of linear filtering similar to a frequency domain concept called "convolution".

$$
\begin{aligned}
& R=w_{1} z_{1}+w_{2} z_{2}+\cdots+w_{m w} z_{m}=\sum_{i=1} w_{i} z_{i} \\
& R=w_{1} z_{1}+w_{2} z_{2}+\cdots+w_{9} z_{9}=\sum_{i=1}^{g} w_{i} z_{i}
\end{aligned}
$$

Where the w's are mask coefficients, the z's are the value of the image gray levels corresponding to those coefficients. Nonlinear spatial filters are also operated on neighborhoods, and the mechanics of sliding a mask part of an image are the same as was just outlined. The filtering operation is based conditionally on the values of the pixels in the neighborhood under consideration. Smoothing filters [5] are used for blurring and for noise reduction. Blurring is used in preprocessing steps, such as removal of small details from an image prior to object extraction, and bridging of small gaps in lines or curves. Noise reduction can be accomplished by blurring. There are two way of smoothing spatial filters. Smoothing Linear Filters and Order-Statistics Filters. Linear spatial filter is simply the average of the pixels contained in the neighborhood of the filter mask sometimes called "averaging filters". The idea is replacing the value of every pixel in an image by the average of the gray levels in the neighborhood defined by the filter mask. Order-statistics filters are nonlinear spatial filters [4] whose response is based on ordering (ranking) the pixels contained in the image area encompassed by the filter, and then replacing the value of the center pixel with the value determined by the ranking result.

\section{IMAGE NOISE MODEL}

An image usually contains departures from the ideal signal that would be produced by general model of the signal production process. Such departures are referred to as noise. Noise arises as a result of un-modeled processes available on in the production and capture of the actual signal. It is not part of the ideal signal and may be caused by a wide range of sources, e.g. variations in the detector sensitivity, environmental variations, the discrete nature of radiation, transmission or quantization errors, etc. It is also possible to treat irrelevant scene details as if they are image noise (e.g. surface reflectance textures). The characteristics of noise depend on its source, as does the operator which best reduces its effects. Deliberately corrupting an image with noise allows us to test the resistance of an image processing operator to noise and assess the performance of various noise filters. Noise can generally be grouped into two classes: (i) 
independent noise, and (ii) noise which is dependent on the image data. Image independent noise can often be described by an additive noise model, where the traced image $f(x, y)$ is the sum of the original image $s(x, y)$ and the noise $n(x, y)$ : i.e. $f(x, y)=s(x, y)+n(x, y)$. In several cases, additive noise is equally distributed over the frequency domain (i.e. white noise), while an image contains generally low frequency information. Hence, the noise is dominant for high frequencies and its effects can be reduced using some kind of low-pass filter. This can be done either with a frequency filter or with a spatial filter [8][9]. (Often a spatial filter is preferable, as it is computationally less expensive than a frequency filter.) In the second case of data dependent noise (e.g. arising when monochromatic radiation is scattered from a surface whose roughness is of the order of a wavelength, causing wave interference which results in image speckle), it is possible to model noise with a multiplicative, or non-linear model. These models are mathematically more complicated. Hence, the noise can be assumed as data independent. Detector Noise [7] is a kind of noise which occurs in all traced images to a certain extent is detector noise. This kind of noise is due to the discrete nature of radiation, i.e. the fact that each imaging system is recording an image by counting photons. Allowing some assumptions (which are valid for many applications) this noise can be modeled with an independent, additive model, where the noise $n(x, y)$ has a zero-mean.

Gaussian distribution described by its standard deviation $(\sigma)$, or variance. This means that each pixel in the noisy image is the sum of the true pixel value and a random Gaussian distributed noise value [6]. It is difficult to propose a general mathematical model for the effect of the abrasion of the film causing the scratches due to the high number of variables that are involved in the process. However, it is possible to make some physical and geometrical considerations regarding the brightness, thickness, and vertical extent of the line. Line scratches can be characterized as follows: (i) they present a considerable higher or lower luminance than their neighborhoods; (ii) they tend to extend over most of the vertical length of the image frame and are not curved; and (iii) they are quite narrow, with widths no larger than 10 pixels for video images. These features can be used to define a model. The degraded image model considered is

$a(x, y)=I(x, y)(I-b(x, y))+b(x, y) c(x, y)$

where $I(x, y)$ is the pixel intensity of the uncorrupted signal, $b(x, y)$ is a detection variable which is set to 1 whenever pixels are corrupted and 0 otherwise, $c(x, y)$ is the observed intensity in the corrupted region. This model is applied in this work to images degraded by impulses, strip lines, drop lines, band missing, and blotches.

If $b(x, y)=0$ then

$a\left(x_{w} y\right)=I\left(x_{w} y\right)(1-0)+0 . c\left(x_{v} y\right)=I\left(x_{v} y\right)$ where $I(x, y)$ is the original pixel value (uncorrupted pixel). If $b(x, y)=0$ then

$a(x, y)=I(x, y)(1-1)+1, c(x, y)=c(x, y)$

where $c(x, y)$ is the original pixel value (uncorrupted pixel). Assume that each pixel at $(x, y)$ is corrupted by an impulse with probability $\mathrm{p}$ independent of whether other pixels are corrupted or not. For images corrupted by a negative or positive impulse, the impulse corrupted pixel $e(x, y)$ takes on the minimum pixel value smin with probability $p$, or $s(x, y)$ the maximum pixel value smax with probability 1-p. The image corrupted by blotches or scratches (impulsive) can be now modeled as

$c(x, y)=e(x, y)$ with $p s(x, y)$ with $1-p$

This, in fact, is the model that describes impulse noise in the literature. However, the existing impulse filtering algorithms do not effectively remove clotches and scratches. In Section 3, an adaptive length median/mean filter algorithm is developed that removes blotches, scratches effectively along with impulse noise.

\section{FILTERING METHODS}

In this section, we provide the definitions of some linear and non-linear image filters. The expression given below can be used to define image processing function in the spatial domain.

$$
f(x, y)=\gamma(g(s, t))(9
$$

where $\gamma$ is the transformation function, $\mathrm{g}(\mathrm{s}, \mathrm{t})$ is the pixel value of the point $\mathrm{p}(\mathrm{x}, \mathrm{y})$ of input image, and $\mathrm{f}(\mathrm{x}, \mathrm{y})$ is the pixel value of the corresponding point of the processed image.

\subsection{Arithmetic mean filter}

$\mathrm{S}_{\mathrm{xy}}$ represent the set of coordinates in mask $\mathrm{m} \mathrm{X} \mathrm{n}$, centered at a point $(\mathrm{x}, \mathrm{y})$. This filter takes input as a corrupted image to calculate it's average value in the area defined by $S_{x y}$. This filter calculate arithmetic mean of restored image $f$ at point $(\mathrm{x}, \mathrm{y})$ using the pixel in the area defined by $\mathrm{S}_{\mathrm{xy}}$

$$
f(x, y)=\frac{1}{m x} \sum_{[a t]] \in S x y} g(s, t)
$$

This operation can be implemented using a spatial filter of $\mathrm{m}$ $\mathrm{X} n$ in which all coefficients have value $1 / \mathrm{mn}$. A mean filter smooths local variations in an image, and noise is reduced as a result of blurring.

\subsection{Geometric mean filter}

The given expression can be used to restore an image

$f\left(x_{w} y\right)=\left[\prod_{\left(g_{w} t\right) \in s_{x y}} g\left(s_{v} t\right)\right]^{\frac{1}{\operatorname{man}}}$

The product of the pixels in the mask provides restored pixel by raising the power $1 / \mathrm{mn}$. It provides more smoothing 
compare than arithmetic mean filter, but loss image details in less.

\subsection{Harmonic mean filter}

The expression for Harmonic mean filter is

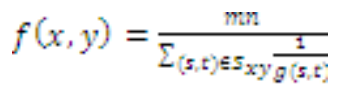

It works well for gaussian noise.

\subsection{Contraharmonic mean filter}

The expression for contraharmonic mean filter is

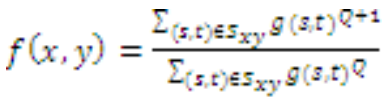

where $\mathrm{Q}$ is order of the filter. Q might have values of 0,1 and -1 .

\subsection{Median filter (MF)}

The best-known order-statistic filter in digital image processing is the median filter. It is a useful tool for reducing salt-and-pepper noise in an image. The median filter [2] plays a key role in image processing and vision. In median filter, the pixel value of a point $p$ is replaced by the median of pixel value of 8-neighborhood of a point ' $p$ '. The operation of this filter can be expressed as:

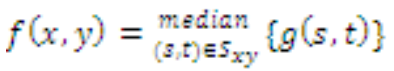

The median filter is popular because of its demonstrated ability to reduce random impulsive noise without blurring edges as much as a comparable linear lowpass filter. However, it often fails to perform well as linear filters in providing sufficient smoothing of nonimpulsive noise components such as additive Gaussian noise [3]. One of the main disadvantages of the basic median filter is that it is location-invariant in nature, and thus also tends to alter the pixels not disturbed by noise.

\subsection{Max filter}

Max filters are very useful for finding the brightest points in an image.

$$
f(x, y)=\max _{[a t] \in S_{x y}} \quad\{g(s, t) \quad(15)
$$

Although the median filter is by far the order-statistic filter most used in image processing, it is by no means the only one. In max filter [1] each output pixel value can be calculated by selecting maximum gray level value of $\mathrm{N}_{8}(p)$. This filter is used for removing the pepper noise from the image. It is also proposed for Gaussian noise removal from the medical image.

\subsection{Min filter}

Algorithm of min filter is implemented from the following expression

$f\left(x_{w} y\right)=\min _{1(a, t) \in s_{x y}}\left\{g\left(s_{v} t\right)\right)$

Min filter [1] recognizes the darkest pixels gray value and retains it by performing min operation. In min filter each output pixel value can be calculated by selecting minimum gray level value of $\mathrm{N}_{8}(\mathrm{p})$. It is used to remove the salt noise from the image. Salt noise has very high values in images. It is also proposed for Gaussian noise removal from the medical image.

\section{RESULTS AND DISCUSSION}

An attempt is made to evaluate the performance of the four linear and non-linear filters used for removing noise from the image. Experimental setup, the images are processed using the Tool Matlab. Comparisons of different filters are done by calculating the Mean Square Error (MSE) \& Peak Signal to Noise Ratio (PSNR). The values are calculated by the following expression.

$P S N R=20 \log _{10} \frac{256}{\sqrt{\text { MFSE }}}$

Where MSE represents the mean square error of the estimation. For Arithmetic mean filtering the baby image is given as input and after filtering the performance is calculated. The MSE value is 31.2570 and the PSNR value is 66.4307 for Gaussian noise. Using median filter the same image is tested for performance. The MSE \& PSNR values are $39.2189 \& 64.4597$ respectively. The best filter must give its performance high in PSNR value and low MSE value. The results obtained out of the four algorithms for different noise applications are shown in table 1 .

Table 1: PSNR \& MSE value

\begin{tabular}{|l|c|c|}
\hline \multicolumn{1}{|c|}{ Method } & PSNR & MSE \\
& value & value \\
\hline $\begin{array}{l}\text { Arithmetic } \\
\text { mean }\end{array}$ & 66.4307 & 31.2570 \\
\hline Median & 64.4597 & 39.2189 \\
\hline Max & 41.5143 & 550.5072 \\
\hline Min & 41.7461 & 536.0123 \\
\hline
\end{tabular}




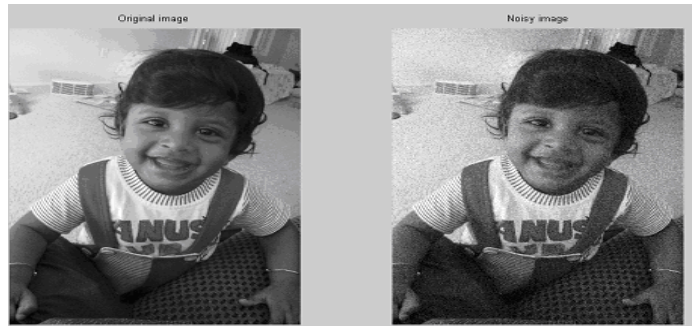

Figure 1 (a) Original image (b) Noisy image

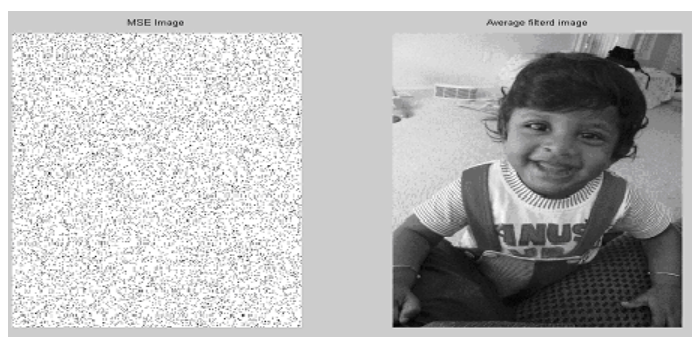

Figure 2 (a) MSE image (b) Average filtered image

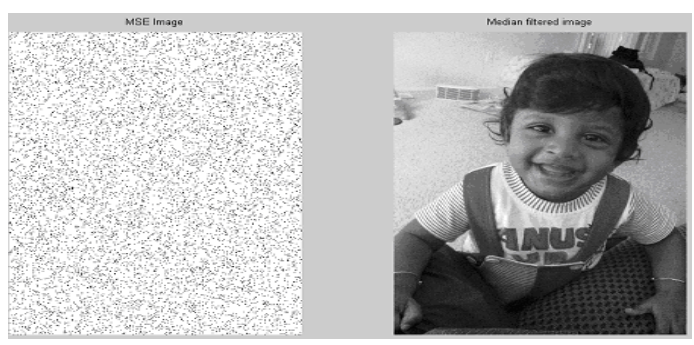

Figure 3 (a) MSE image (b) Median filtered image

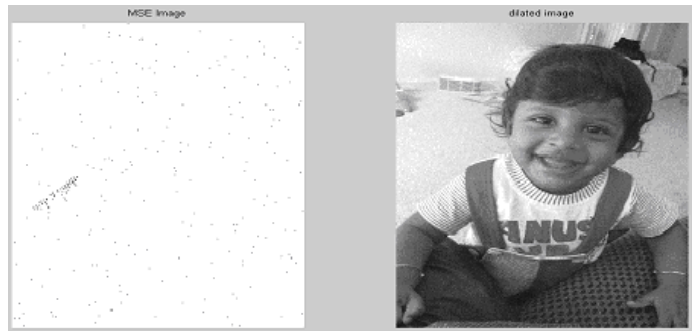

Figure 4 (a) MSE image (b) Diated image

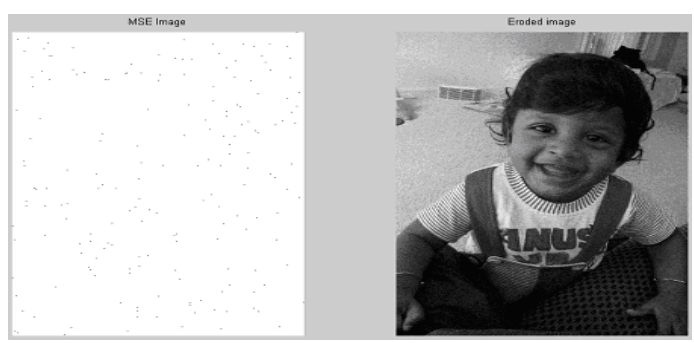

Figure 5 (a) MSE image (b) Eroded image

\section{CONCLUSIONS}

In this work, we have taken various spatial filtering techniques for removal of Gaussian noise from a given image. To demonstrate the performance of the proposed techniques, the experiments have been conducted. The performance of Gaussian noise removing spatial filtering techniques is measured using quantitative performance measures such as MSE and PSNR. The experimental results indicate that the one of the filter, Arithmetic mean Filter performs significantly better than many other existing techniques and it gives the best results after successive iterations. The proposed method is simple and easy to implement.

\section{REFERENCES:}

[ 1] H. Hu and G. de Haan. 2006. Classification-based hybrid filters for image processing. Proc. SPIE, Visual Communications and Image Processing. 6077, 607711.1-607711.10.

[2] Hakan Güray Senel, Richard Alan Peters and Benoit Dawant. 2002. Topological Median Filter. IEEE Trans on Image Processing. 11(2): 89-104.

[3] How-Lung Eng, Student Member, IEEE, and KaiKuang Ma. 2001. Noise Adaptive Soft-Switching Median Filter : IEEE Transactions On Image Processing, VOL. 10, NO. 2.

[4] Ioannis Pitas, Anastasias N Venetsanopoulos. 1990. Nonlinear Digital Filters: Principles and Applications. NJ: Springer Publisher.

[5] J. W. Tukey. 1974. Nonlinear (nonsuperposable) methods for smoothing data. In: Proc. Congr. Rec. EASCOM '74. pp. 673-681.

[6] Klaus Rank and Rolf Unbehauen. 1992. An Adaptive Recursive 2-D Filter for Removal of Gaussian Noise in Images. IEEE Transactions on Image Processing: 431-436.

[7] Mamta Juneja and Rajni Mohana. 2009. An Improved Adaptive Median Filtering Method for Impulse Noise Detection. International Journal of Recent Trends in Engineering. 1(1): 274-278.

[8] P. Kuosmanen and J. Astola, "Fundamentals of Nonlinear Digital Filtering," CRC Press Inc.,Boca Raton, 1998.

[ 9] R. Gonzalez and R. Woods. 1992. Digital Image Processing. Adison -Wesley, New York.

[10] S. Peng and L. Lucke. 1995. A hybrid filter for image enhancement. Proc. of International Conference on Image Processing (ICIP). 1: 163166.

[11] Yanchun Wang, Dequn Liang, Heng Ma and Yan Wang. 2006. An Algorithm for Image Denoising Based on Mixed Filter. Proceedings of the $6^{\text {th }}$ World Congress on Intelligent Control and Automation. June 21-23. pp. 9690-9693. 